\title{
Etude de la qualité rédactionnelle des bulletins d'analyses biologiques au CHU Yalgado Ouédraogo, Burkina Faso
}

\author{
Abdourahamane YACOUBA ${ }^{1 *}$, Kady KIELLO ${ }^{1}$, T.C.R. Alice KIBA-KOUMARE ${ }^{1,2}$, \\ Elie $\mathrm{KABRE}^{1,2}$ et Jean SAKANDE ${ }^{1,2}$ \\ ${ }^{1}$ Service de Biochimie, Centre Hospitalier Universitaire Yalgado Ouédraogo ; 03 BP 7022 Ouaga 03, \\ Ouagadougou, Burkina Faso. \\ ${ }^{2}$ Unité de Formation et des Recherche en Sciences de la Santé, Université Ouaga I Pr Joseph KI-ZERBO ; 03 \\ B.P. 7021 Ouaga 03, Ouagadougou, Burkina Faso. \\ *Auteur correspondant ; E-mail : abdourahamaneyacouba@yahoo.fr; Tel : +22799185000.
}

\section{RESUME}

La non-conformité des bulletins d'analyses biologiques (BAB) rend difficile la réalisation et l'interprétation des résultats des examens biologiques. Elle compromet de ce fait l'utilisation optimale et rationnelle de l'outil d'aide au diagnostic que constituent les laboratoires. L'objectif de cette étude était d'évaluer la conformité des bulletins d'analyses biologiques prescrits et leur impact économique au Centre Hospitalier Yalgado Ouédraogo (CHU-YO), Burkina Faso. Il s'est agi d'une étude transversale descriptive réalisée de décembre 2016 à février 2017 dans le département de laboratoire du CHU-YO. Les informations portées sur chaque BAB et les coûts des examens prescrits sur ces bulletins ont été étudiés. Au total, 1683 BAB provenant de 10 services cliniques, ont été inclus dans l'étude. Sur les 1683 BAB, 71 (4,2\%) étaient conformes aux règles de bonne prescription médicale. Le coût moyen des examens prescrits sur des $\mathrm{BAB}$ non conformes (9,2 \$US) était significativement plus élevé par rapport à ceux prescrits sur des BAB conformes $(7,12$ \$US) (p $<0,05)$. La qualité rédactionnelle des BAB est faible au Burkina Faso. Les BAB non conformes ont un impact réel sur le revenu du ménage et par conséquent sur l'économie du pays.

(C) 2019 International Formulae Group. All rights reserved.

Mots clés: Conformité, bulletins d'analyses biologiques, impact économique, Burkina Faso.

\section{Writing quality of the laboratory request forms at Yalgado Ouédraogo teaching hospital, Burkina Faso}

\begin{abstract}
The non-conformity of the laboratory requesting forms (LRF) makes it difficult to produce and interpret the results of the biological test; it compromises the optimal and rational use of the laboratory diagnostic tool. The aim of this study was to evaluate the conformity of the laboratory requisition forms prescribed and their economic impact at Yalgado Ouédraogo teaching hospital (CHU-YO), Burkina Faso. Cross-sectional and descriptive study was conducted between December 2016 and February 2017 in the department of laboratory, CHU-YO. The information provided of each request form and the cost of investigation required on each one were recorded and analyzed. A total of 1683 LRF from 10 clinicals services
\end{abstract}


were included. Of the 1683 LFR, 71 (4.2\%) complied with the rules of good medical prescription. There is a significant difference between the average cost of tests prescribed on non-compliant LRF (9.2 \$US) and those prescribed on compliant LRF (7.12 \$US) ( $\mathrm{p}<0.05)$. The drafting quality of LRF is low in Burkina Faso. The occurrence of errors in the writing of the LRF has a real impact on the household income and consequently on the country's economy.

(C) 2019 International Formulae Group. All rights reserved.

Keywords: Conformity, laboratory requesting forms, economic impact, Burkina Faso.

\section{INTRODUCTION}

Le bulletin d'analyses biologiques (BAB) est un document rédigé par un praticien, remis au malade pour lequel l'élément à prescrire est une investigation paraclinique en biologie médicale (Adeoti et al., 2004). C'est une ordonnance médicale à visée diagnostique comportant un ensemble d'informations sur le prescripteur, le patient et le prélèvement. Aussi les examens biologiques demandés, le service demandeur ainsi qu'un ensemble d'informations en rapport avec l'hypothèse diagnostique envisagée et le traitement en cours doivent être correctement libellés (Adeoti et al., 2004). Ces informations sont utiles pour la bonne exécution des analyses et pour l'interprétation des résultats.

Le laboratoire d'analyses médicales joue un rôle central dans la prise en charge des patients. Selon certains auteurs, 60 à 70\% des décisions liées à l'hospitalisation, aux médicaments prescrits et à l'autorisation de sortie des patients, étaient influencées par un résultat d'analyses biologiques (Plebani, 2006 ; Nutt et al., 2008). Cette dépendance de la prise en charge des patients vis à vis des résultats d'analyses biologiques souligne la nécessité pour les prescripteurs qui sollicitent une analyse complémentaire, de rédiger la requête en mentionnant les informations définies par les règles des bonnes pratiques rédactionnelles d'un BAB (Adeoti et al., 2004 ; Szymanowicz, 2010). L'absence d'une de ces informations ou la survenue des erreurs lors de la rédaction des $\mathrm{BAB}$ peut affecter la qualité d'exécution des examens, rallonger les délais de transmission des résultats et occasionner des reprises des mêmes examens pour les mêmes patients, entraînant des coûts supplémentaires inutiles (Olayemi et Asiamah-Broni, 2011; Singh et al., 2015; Toshniwal et al., 2017). Dès lors, la conformité des BAB est l'un des principaux critères de qualité de la phase pré-analytique et constitue une notion qui met en jeu la responsabilité du biologiste qui doit veiller à sa bonne exécution.

Au Burkina Faso, très peu d'études ont exploré la conformité des BAB pourtant source de nombreuses critiques. Ainsi, un état des lieux s'imposait.

L'objectif de notre étude était d'évaluer la conformité des bulletins d'analyses biologiques prescrits au centre hospitalier universitaire Yalgado Ouédraogo (CHU-YO) et leur impact économique.

\section{MATERIEL ET METHODES \\ Cadre d'étude}

Cette étude s'est déroulée dans le département des laboratoires du CHU-YO, principal centre hospitalier chargé de la formation des médecins aux Burkina Faso. Les services concernés étaient les suivants: service de bactériologie-virologie, de biochimie, d'hématologie et de parasitologiemycologie.

\section{Type et période de l'étude}

Il s'est agi d'une étude transversale descriptive, réalisée de décembre 2016 à février 2017.

\section{Méthode d'échantillonnage et de collecte des données}

L'échantillonnage utilisé était de type consécutif non exhaustif. Tous les BAB prescrits dans le cadre de la gratuité des soins et qui répondaient aux critères d'inclusion ont constitué l'échantillon de l'étude. Le choix 
des bulletins de la gratuité des soins était motivé par des raisons pratiques. En effet, ceux-ci sont rédigés sur des papiers souches faits de plusieurs copies dont une copie était gardée au laboratoire pour le besoin du service de comptabilité. Cette copie du BAB a été utilisée pour la collecte des données de l'étude facilitant ainsi ce processus.

Pour chaque $\mathrm{BAB}$, les informations présentées dans le Tableau 1 ont été recherchées et enregistrées sur une fiche de collecte des données, créée à cet effet. Chaque fiche de collecte correspond à un bulletin et chaque bulletin à un patient. Un BAB était déclaré conforme lorsqu'il portait tous les renseignements suivants : les informations sur le patient (identité, âge, sexe), le service demandeur, les informations sur le prescripteur (identité, signature, qualification et cachet), les renseignements cliniques et thérapeutiques, la date de prescription et la nature de l'échantillon (sang, urine, pus, etc.). Cette définition tient compte des recommandations sur les règles de bonnes pratiques rédactionnelles des BAB (Adeoti et al., 2004 ; Szymanowicz, 2010).

\section{Critères d'inclusion}

Tous les BAB prescrits dans le cadre de la gratuité des soins reçus au niveau des 4 services concernés par l'étude au cours de la période d'enquête ont été inclus dans l'étude.

\section{Variables de l'étude}

Les informations présentées dans le Tableau 1 ont constitué les variables de l'étude.

\section{Saisie et analyse des données}

Les données recueillies ont été saisies sur Microsoft ${ }^{\circledR}$ Excel 2013 puis analysées avec RStudio (Version 0.99.903). Le test statistique de $\mathrm{Chi}^{2}$ a été utilisé pour les comparaisons des proportions entre deux variables. Le test $t$ de Student a été utilisé pour déterminer les coûts moyens des $\mathrm{BAB}$ en fonction de la conformité. Le coût global d'un bulletin correspond à la somme des coûts de tous les examens demandés sur le même bulletin. Le seuil de signification statistique a été fixé à $p<0,05$.

\section{Considérations éthiques}

L'accord des chefs des services des laboratoires concernés a été demandé et obtenu avant la réalisation de cette étude. Aussi, l'anonymat des patients et des prescripteurs a été respecté.

Tableau 1: Informations recherchées sur chaque bulletin d'analyse biologique.

\begin{tabular}{lll}
\hline \multicolumn{1}{c}{ Informations } & \\
\hline Sur le patient & Sur l'examen / prélèvement & Sur le prescripteur \\
\hline Identité* & Libellé exacte de(s) examen(s) demandé(s) & Identité \\
Age & Date de prescription & Qualification \\
Sexe & Nature de prélèvement & Signature \\
Renseignements cliniques** & Date de prélèvement & Cachet \\
& Heure du prélèvement & Contact \\
\hline $\begin{array}{l}* * \text { Nom et prénom du patient. } \\
* \text { symptômes, diagnostic, traitement en cours, état physiologique. }\end{array}$
\end{tabular}




\section{RESULTATS}

$\mathrm{Au}$ total, $1683 \mathrm{BAB}$ provenant de 10 services cliniques ont été inclus dans l'étude. Sur les 1683 BAB, 3506 examens ont été demandés, parmi lesquels, $1721(49,1 \%)$ de biochimie, 1143 (32,6\%) d'hématologie, 458 $(13,1 \%)$ de bactériologie-virologie et 184 $(5,2 \%)$ de parasitologie-mycologie.

La majorité des $\mathrm{BAB}$ ont été rédigés par les stagiaires en médecine $(71,4 \%)$. Les BAB provenant du service de pédiatrie et du service de gynécologie-obstétrique étaient les plus représentés avec respectivement 49,5\% $(n=833)$ et $30,2 \%(n=509)$. Le laboratoire ayant reçu le plus grand nombre des BAB était celui d'hématologie, 695 (41,3\%), suivi par celui de biochimie, $500(29,7 \%)$.

L'analyse des $\mathrm{BAB}$ a révélé que les informations les plus fréquentes sur les $\mathrm{BAB}$ étaient l'identité du patient $(99,8 \%)$, la date de prescription $(99,3 \%)$ et l'âge du patient
(96,7\%). Par contre, 99,0\% des BAB ne portaient pas le contact du prescripteur et aucun $(0 \%)$ BAB ne présentait la date et l'heure du prélèvement (Tableau 2).

Sur les 1683 BAB, 71 (4,2\%) étaient conformes aux règles de bonne prescription médicale. Parmi les 71 BAB conformes, $18,3 \%$ étaient prescrits par des médecins titulaires contre $81,7 \%$ par des stagiaires en médecine $(\mathrm{p}=0,4158)$ (Tableau 3$)$.

Le coût global des examens prescrits sur l'ensemble des BAB était de 15180.15 \$US. La répartition du coût selon la conformité a montré que le coût moyen des examens prescrits sur les BAB non conformes (9,2 \$US) était significativement plus élevé que celui des examens prescrits sur les $\mathrm{BAB}$ conformes (7,12 \$US) (p < $2,2.10^{-16}$; IC95\% (\$US) = 1,71 à 24,81) (Tableau 4).

Tableau 2: Informations requises sur les bulletins d'analyses biologiques et fréquences de leur notification.

\begin{tabular}{|c|c|c|c|c|}
\hline \multirow{3}{*}{ Critères } & \multicolumn{4}{|c|}{ Existence } \\
\hline & \multicolumn{2}{|c|}{ Oui } & \multicolumn{2}{|c|}{ Non } \\
\hline & Effectifs & $\%$ & Effectifs & $\%$ \\
\hline Nom et prénom du patient & 1680 & 99,8 & 3 & 0,2 \\
\hline Age du patient & 1627 & 96,7 & 56 & 3,3 \\
\hline Sexe du patient & 1587 & 94,3 & 96 & 5,7 \\
\hline Identité du prescripteur & 1584 & 94,1 & 99 & 5,9 \\
\hline Signature du prescripteur & 1585 & 94,2 & 98 & 5,8 \\
\hline Qualification du prescripteur & 1562 & 92,8 & 121 & 7,2 \\
\hline Cachet du prescripteur & 743 & 44,1 & 940 & 55,9 \\
\hline Contact du prescripteur & 17 & 1,0 & 1666 & 99,0 \\
\hline Date de prescription & 1671 & 99,3 & 12 & 0,7 \\
\hline Renseignements cliniques & 683 & 40,6 & 1000 & 59,4 \\
\hline Nature de l'échantillon & 285 & 16,9 & 1398 & 83,1 \\
\hline Date de prélèvement & 0 & 0 & 1683 & 100,0 \\
\hline Heure du prélèvement & 0 & 0 & 1683 & 100,0 \\
\hline Service demandeur & 1391 & 82,7 & 292 & 17,3 \\
\hline
\end{tabular}


Tableau 3: Conformité des bulletins en fonction de la qualification des prescripteurs.

\begin{tabular}{lccc}
\hline Qualification du prescripteur & \multicolumn{2}{c}{ Prescriptions conformes } & \multirow{2}{*}{ p value } \\
\cline { 2 - 3 } & Non $(\%)$ & Oui $(\%)$ & \\
\hline Médecins titulaires & $346(23,2)$ & $13(18,3)$ & \\
Stagiaires en médecine & $1145(76,8)$ & $58(81,7)$ & 0,4158 \\
\hline Total & $1491(100,0)$ & $71(100,0)$ & \\
\hline
\end{tabular}

Tableau 4: Coûts des examens selon la conformité.

\begin{tabular}{lccc}
\hline & \multicolumn{2}{c}{ Prescriptions conformes } & \multirow{2}{*}{ Total (\$ US) } \\
\cline { 2 - 3 } & Non & Oui & \\
\hline Coût global (\$ US) & 14674,98 & 505,17 & 15180,15 \\
Coût moyen (\$ US) & 9,2 & 7,12 & 16,32 \\
\hline
\end{tabular}

p-value $<2,2.10^{-16^{*}} ;$ IC95\% (\$US) $=1,71$ à 24,81

*Significatif ; IC95\% = intervalle de confiance à 95\% ; \$US = Dollars United States.

\section{DISCUSSION}

Les BAB inclus dans cette étude sont ceux prescrits dans le cadre de la gratuité des soins offerts aux enfants de moins de 5 ans et à la femme enceinte. Il y a donc nécessairement une sélection des services demandeurs (et donc des prescripteurs). Cependant, ce biais peut être relativisé. En effet, si les services de pédiatrie et gynécologie-obstétrique constituent en majorité les portes d'entrées dans la gratuité des soins, tous les autres services peuvent être sollicités, en témoigne le nombre $(\mathrm{n}=10)$ des services demandeurs dans la présente étude.

Dans cette étude, les stagiaires en médecine représentaient $71,4 \%$ des prescripteurs et les médecins titulaires $21,3 \%$ des prescripteurs. Ceci pourrait s'expliquer par le nombre important des stagiaires en médecine dans les différents services du fait que le CHU-YO représente le principal centre hospitalier universitaire chargé de la formation pratique des médecins au Burkina
Faso. Aussi, les stagiaires prescrivent comme les titulaires quel que soit le service clinique du CHU-YO. L'orthodoxie de la prescription médicale devrait être appliquée par les médecins titulaires en charge de l'encadrement des stagiaires dans le contexte d'un centre hospitalier universitaire, si l'on veut améliorer la qualité de la prescription des futurs cliniciens.

Les informations les plus notées sur les BAB étaient l'identité du patient $(99,8 \%)$, la date de prescription (99,3\%), l'âge (96,7\%) et le sexe du patient $(94,3 \%)$. Ces résultats étaient proches de ceux observés dans la littérature (Nutt et al., 2008; Singh et al., 2015 ; Toshniwal et al., 2017). Par contre, l'identité, la qualification et le contact du prescripteur n'étaient pas précisés respectivement dans $5,9 \% ; 7,2 \%$ et $99,0 \%$ des BAB de cette étude. Ces informations sont des éléments d'identification professionnelle du prescripteur qui confèrent à la prescription un sérieux suffisant pour une meilleure 
exécution par le laboratoire et permettent au biologiste de savoir si le prescripteur est qualifié et/ou autorisé à prescrire (Adeoti et al., 2004). En outre, elles permettent d'adapter les commentaires joints aux résultats pour une meilleure compréhension du prescripteur. L'absence de ces informations complémentaires limite la communication entre biologiste et clinicien en cas de besoin de renseignements complémentaires sur le patient ou la transmission des résultats critiques qui méritent une prise en charge immédiate. Nutt et al. (2008) ont rapporté 30 $(19,9 \%)$ cas des résultats critiques (dont 17 cas d'hyperkaliémie comprise entre $6-8,7$ $\mathrm{mmol} / \mathrm{L}$ ) pour lesquels les $\mathrm{BAB}$ ne présentaient pas de contact du prescripteur. Parmi eux, 6 (20\%) BAB ne portaient aucune information sur les prescripteurs.

$\mathrm{L}$ 'analyse des $\mathrm{BAB}$ a révélé qu'aucun $(0 \%)$ ne présentait la date et l'heure du prélèvement. Ces dernières sont pourtant capitales lors des certaines analyses; par exemple, lors de la réalisation du spermogramme, la mobilité à la première heure des spermatozoïdes est un paramètre important, faisant de l'heure du prélèvement un élément déterminant pour la qualité de l'interprétation des résultats.

La nature de l'échantillon (sang, urine, liquide cérébro-spinal, etc.) n'a pas été notée sur $83,1 \%$ des BAB de cette étude. Des résultats proches aux nôtres ont été rapportés dans la littérature (Gyawali et al., 2012; Oladeinde et al., 2012). La mention de la nature de l'échantillon est importante en cas de prélèvement des liquides biologiques sanguinolents (urine, ascite, liquide pleural, liquide cérébro-spinal, etc.) qui peuvent être confondus avec du sang et cela peut entrainer l'utilisation des valeurs de référence inappropriées pour l'interprétation des résultats (Nutt et al., 2008).

Les renseignements cliniques et thérapeutiques étaient absents sur $59,4 \%$ des $\mathrm{BAB}$ de cette étude. Ces résultats étaient en accord avec ceux observés dans d'autres pays d'Afrique et d'Asie (Olayemi et AsiamahBroni, 2011; Oyedeji et al., 2015 ; Toshniwal et al., 2017). La mention des renseignements cliniques et thérapeutiques peut aider le biologiste dans le choix de la technique à utiliser ou l'amener à déconseiller une investigation coûteuse pour en suggérer une autre plus adéquate. Par exemple, le diagnostic précoce de la grossesse se fait sur un échantillon sanguin avec un appareillage utilisant une méthode immunoenzymatique alors que le diagnostic tardif se fait simplement sur un échantillon urinaire à l'aide d'une technique manuelle utilisant des bandelettes réactives. De même, les renseignements cliniques sont indispensables pour l'interprétation des résultats de laboratoire. En effet, plusieurs types d'échantillons peuvent être utilisés pour réaliser un même examen et le plus indiqué est choisi en fonction du cas clinique ; c'est le cas de la fièvre typhoïde où la recherche de l'agent causal est réalisée selon le stade de la maladie, dans les selles (coproculture) ou le sang (hémoculture). Il paraît donc évident qu'il est bien de noter les renseignements cliniques, mais faudrait-il encore qu'ils soient adaptés à l'examen demandé. Sakandé et al. (2003) ont relevé une inadéquation entre les renseignements cliniques fournis et les examens demandés.

Par ailleurs, la mention des renseignements cliniques et thérapeutiques soulève une autre question relative au secret professionnel. En effet, dans notre contexte, si les biologistes sont soumis aux mêmes règles de l'éthique et de la déontologie médicale que les prescripteurs, il n'en est pas le cas pour les accompagnateurs des patients (parents, tuteur, conjoint, amis, etc.) à qui l'on remet souvent les $\mathrm{BAB}$ portant des renseignements cliniques et thérapeutiques sur le malade afin d'aller les régulariser au niveau de la caisse de l'hôpital. Ce faisant, les accompagnateurs ont accès à des informations qui relèvent du secret professionnel que le concerné pourrait ne pas vouloir partager avec eux.

Les résultats de cette étude ont montré que sur un total de $1683 \mathrm{BAB}$, seulement 71 $(4,2 \%)$ étaient jugés conformes aux règles de bonne prescription médicale. Ces résultats confirment que la qualité rédactionnelle des $\mathrm{BAB}$ est faible au Burkina Faso. La non 
qualité de la prescription rend difficile la réalisation et l'interprétation des résultats des examens biologiques. Ceci compromet l'utilisation optimale et rationnelle de l'outil d'aide au diagnostic que constituent les laboratoires. Dogethera et al. (2016) proposent l'utilisation d'un logiciel pour la saisie des prescriptions d'analyses biologiques afin d'améliorer leurs qualités rédactionnelles. Cependant, cette perspective d'emploi de logiciel ne nous paraît pas envisageable à court terme dans notre contexte.

Les coûts des examens prescrits selon leurs conformités ont été déterminés en définissant le coût global d'un bulletin comme la somme des coûts de tous les examens demandés sur le même bulletin. Ainsi, le coût des examens prescrits sur les bulletins non conformes représentait 96, 7\% (14674,98 \$US) du coût global des examens. Le coût moyen des examens prescrits sur les $\mathrm{BAB}$ non conformes (9,2 \$US) était significativement plus élevé que celui des examens prescrits sur les BAB conformes (7,12 \$US). Le revenu national brut par habitant au Burkina Faso étant de 620 \$US en 2016 (Banque mondiale, 2016), il ressort de ces résultats que la survenue des erreurs lors de la rédaction du $\mathrm{BAB}$ a un impact réel sur le revenu du ménage et, par conséquent, sur l'économie du pays.

\section{Conclusion}

La conformité des $\mathrm{BAB}$ prescrits au CHU-YO a été évaluée selon les recommandations sur les règles de bonnes pratiques rédactionnelles des $\mathrm{BAB}$. Les coûts des examens prescrits selon leurs conformités ont été également déterminés pour évaluer le poids économique de la qualité rédactionnelle des BAB. Les résultats de cette étude confirment que la qualité rédactionnelle des $\mathrm{BAB}$ est faible au Burkina Faso. La survenue des erreurs lors de la rédaction du $\mathrm{BAB}$ a un impact réel sur le revenu du ménage et, par conséquent, sur l'économie du pays. Une attitude de fermeté est nécessaire au niveau du laboratoire pour les respects des critères de conformité en rejetant tout bulletin d'analyses biologiques qui ne satisfait pas à ces conditions. Aussi envisageons-nous d'induire l'amélioration de la prescription médicale par un retour des résultats de cette étude vers les praticiens et par des évaluations similaires réalisées de manière régulière.

\section{CONFLIT D'INTERETS}

Les auteurs déclarent qu'ils n'ont aucun conflit d'intérêts.

\section{CONTRIBUTIONS DES AUTEURS}

KK a participé à la collecte et à la saisie des données. AY a contribué à l'analyse des données et à la rédaction du manuscrit. AKK a participé à la relecture du manuscrit. EK et JS ont supervisé le travail.

\section{REMERCIEMENTS}

Les auteurs remercient tous le personnel des laboratoires de bactériologievirologie, de biochimie, d'hématologie et de parasitologie-mycologie pour leur collaboration.

\section{REFERENCES}

Adeoti MF, Silue A, Sawadogo D, Dosso M, Sess ED. 2004. Réflexion sur les bonnes pratiques de la rédaction du bulletin d'analyses de biologie médicale. Immuno-Analyse et Biologie Specialisée, 6(19): 370-373.

Doi:10.1016/j.immbio.2004.08.001.

Banque mondiale: Burkina Faso data 2016. Accessed $07 \quad$ February 2018; https://donnees.banquemondiale.org/pay s/burkina-faso.

Dogethera MA, Muallema YA, Househ M, Saddika B, Khalifa M. 2016. The impact of automating laboratory request forms on the quality of health care services. $J I P H, \quad$ 9: $749-756 . \quad$ DOI: http://dx.doi.org/10.1016/j.jiph.2016.09. 003.

Gyawali P, Shrestha RK, Bhattarai P, Raut BK, Aryal M, Malla SS. 2012. Evaluation of pre-analytical errors: inadequacies in the completion of laboratory requisition forms. JNAMLS, 
11(1): 43-48.

Nutt L, Zemlin AE, Erasmus RT. 2008. Incomplete laboratory request forms: the extent and impact on critical results at a tertiary hospital in South Africa. Ann Clin Biochem., 45(5): 463-466. DOI: http://dx.doi.org/10.1258/acb.2008.0072 52.

Oladeinde BH, Omoregie R, Osakue EO, Onifade AA. 2012. Evaluation of laboratory request forms for incomplete data at a rural tertiary hospital in Nigeria. N. Z. J. Med. Lab. Sci., 66: 39-41.

Olayemi E, Asiamah-Broni R. 2011. Evaluation of request forms submitted to the haematology laboratory in a Ghanaian tertiary hospital. Pan. Afr. Med. J., 8(33). http://www.panafricanmedjournal.com/content/article/8/33/full/.

Oyedeji OA, Ogbenna AA, Iwuala SO. 2015. An audit of request forms submitted in a multidisciplinary diagnostic center in Lagos. Pan. Afr. Med. J., 20(423).
Plebani M. 2006. Errors in clinical laboratories or errors in laboratory medicine? Clin. Chem. Lab. Med., 44(6): 750-759.

DOI: https://doi.org/10.1515/CCLM.2006.123. Sakandé J, Diafouka F, Tiahou G, Sess ED, Yapo AE. 2003. Evaluation économique $\mathrm{du}$ bilan biologique au $\mathrm{CHU}$ de Yopougon: Expérience du laboratoire de biochimie. Mali Médical, 18(1\&2): 6-7.

Singh S, Khatiwada S. 2015. Assessment of biochemistry request forms. Journal of Chitwan Medical College, 5(13): 18-42.

Szymanowicz A. 2010. Analyse interprétative de la norme ISO 15189: aspects préanalytiques. Ann. Biol. Clin., 68(1): 155-162. DOI: 10.1684/abc.2011.0552.

Toshniwal P, Toshniwal S, Jasani J, Shah RM. 2017. Test requisition form- A check point in pre-analytical phase for laboratory errors. Asian. Pac. J. Health Sci., 4(2): 175-182. DOI: 10.21276/apjhs.2017.4.2.29. 